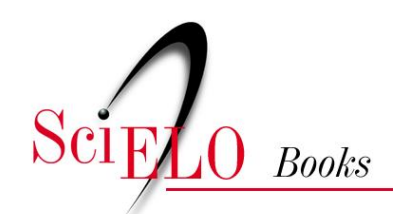

\title{
Desafíos de la formación de profesorado para la educación intercultural bilingüe
}

\author{
María Sol Villagómez R.
}

\section{SciELO Books / SciELO Livros / SciELO Libros}

VILLAGÓMEZ R., M. S. Desafíos de la formación de profesorado para la educación intercultural bilingüe. In: GARCÉS, F., and BRAVO, R., eds. Interculturalidad. Problemáticas y perspectivas diversas [online]. Quito: Editorial Abya-Yala, 2019, pp. 217-240. ISBN: 978-9978-10-497-2. https://doi.org/10.7476/9789978104972.0008.

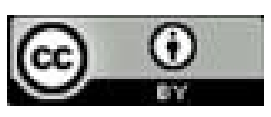

All the contents of this work, except where otherwise noted, is licensed under a Creative Commons Attribution 4.0 International license.

Todo o conteúdo deste trabalho, exceto quando houver ressalva, é publicado sob a licença Creative Commons Atribição 4.0.

Todo el contenido de esta obra, excepto donde se indique lo contrario, está bajo licencia de la licencia $\underline{\text { Creative }}$ Commons Reconocimento 4.0. 


\section{Desafíos de la formación de profesorado para la educación intercultural bilingüe}

María Sol Villagómez R. Carrera de Educación Intercultural Bilingüe- UPS

Desde que sali de casa, traje el viaje de vuelta gravado en mi mano, enterrado en el ombligo, dentro y fuera, así conmigo, mi propia orientación.

(Torquato Neto).

\section{Introducción}

Reflexionar sobre los desafíos de la experiencia de la formación de profesorado en la carrera de educación intercultural bilingüe (EIB) de la Universidad Politécnica Salesiana es siempre un ejercicio provocador, no solo por la particularidad y originalidad del proyecto educativo y sus destinatarios, sino por su permanencia y actividad por más de 25 años. Este trabajo no pretende ser de tipo conclusivo, ni tampoco logra abarcar toda la problemática del campo de estudio de la formación docente; su objetivo es presentar la experiencia de la carrera como un proyecto educativo destinado a la formación de profesorado para la EIB y los desafíos de su puesta en ejecución. La centralidad de la reflexión se encuentra en dos dimensiones: la singularidad del diseño curricular de la carrera y la perspectiva de los actores sobre su implementación. El trabajo se divide en cuatro secciones: en primer lugar, se pone en evidencia la importancia de la creación de la carrera como respuesta a las demandas de los contextos; a continuación, se presenta la trayectoria 
del programa educativo; seguidamente se revisa el currículo de la carrera destacando su especificidad como proyecto educativo de formación docente para la EIB, para finalmente mostrar los desafíos de las concreciones educativas y de la praxis. Los datos presentados, tanto testimoniales, como los que corresponden a la revisión de la currícula han sido tomados de la tesis doctoral de la autora Villagómez (2016).

\section{La necesidad de formar docentes para la EIB}

La institucionalización de la EIB a fines de la década de 1980 fue uno de los logros más importantes de la organización indígena ecuatoriana en lo que respecta a reivindicaciones en el campo de la educación y escolarización de su pueblo. El ingreso de las escuelas indígenas, que hasta el momento operaban sin el reconocimiento del Estado, de manera 'clandestina' -como es el caso del Sistema de Escuelas Indígenas de Cotopaxi (SEIC) - a la jurisdicción intercultural bilingüe, provocaría importantes cambios en la estructura y formas de gestión (Granda, 2018, p. 295). Uno de ellos tiene que ver con las políticas y prácticas docentes (formación, selección y trabajo docente) para la EIB.

Si bien las escuelas interculturales, requerían contar con docentes pertenecientes a las mismas comunidades -elemento central en los modelos educación propia- la institucionalización de la EIB, demandaba algunos requerimientos específicos para el acceso y desempeño de la profesión.

Las exigencias más inmediatas son las de ingreso al magisterio y se relacionarían con la titulación, "ya no era suficiente que los educadores sean comunitarios; estos educadores comunitarios debían acreditar formación docente" (Salesiano A, entrevista, 2014), pero además la formación de estos docentes, no podía, en términos de proyecto educativo asimilarse a la formación convencional de profesorado para las escuelas hispanas ${ }^{1}$, era

1 Hasta el año de 2010, el sistema educativo nacional se encontraba dividido en Sistema de Educación Intercultural Bilingüe, creado en 1988 y el Sistema educativo "hispano" que atendía a población mestiza monolingüe hispanohablante en instituciones educativas o escuelas "hispanas". 
necesario considerar entonces los contextos, los sujetos y sus necesidades formativas y las pedagogías para esa realidad en particular.

Desde estas demandas, se requería contar con espacios de educación formal donde los educadores que se encontraban en ejercicio en las escuelas comunitarias y los nuevos docentes que el SEIB (Sistema de Educación Intercultural Bilingüe) requería para las escuelas interculturales bilingües pudieran profesionalizarse. La intención era "formar un docente al servicio de la comunidad con una perspectiva liberadora, crítica, con fuerte identidad cultural, (...)" (Salesiano A, entrevista, 2014).

\section{Trayecto de la carrera de Educación Intercultural Bilingüe de la UPS}

Como se ha señalado, las demandas de educación superior por parte del pueblo indígena impulsaron la creación del Programa Académico Cotopaxi (PAC), en 1994 para la formación de docentes para la EIB. En 1995, se oficializa el programa, con la creación de la Universidad Politécnica Salesiana (UPS), establecida mediante Ley del Congreso Nacional (Registro Oficial no 499 del 05 de agosto de 1994, ley N 63), una vez que se suscribe el convenio entre la UPS y el SEIC. "Es la universidad la que otorga los títulos académicos” (Farfán, 2008, p. 285).

El PAC, se funda en la parroquia indígena de Zumbahua en la zona del Quilotoa del cantón Pujilí en la provincia de Cotopaxi, convirtiéndose así en la primera y única universidad en el páramo ecuatoriano, las motivaciones de su ubicación geográfica tuvieron que ver con las demandas y necesidades contextuales y educativas del sector y población a la que se atendía:

Nos llevamos la universidad al páramo porque queríamos llegar a nuestros destinatarios, porque considerábamos que el contexto geográfico, económico y cultural es una mediación formativa y porque económicamente era una alternativa accesible a los destinatarios. El 85\% del costo real estaba subsidiado gracias al trabajo del equipo pastoral y del equipo docente. La experiencia se inició y se ha sostenido con sacrificio y me siento orgulloso de ser uno de los docentes pioneros que durante ocho años 
nos hemos mantenido como "educadores de alto nivel" (3500 m.s.n.m.). (carta de Martínez, s.a, dirigida a Delgado; citado por Reyes, 2006, p. 23)

Para los alumnos-maestros que se encontraban en ejercicio de la docencia en las escuelas comunitarias, el PAC constituyó una importante oportunidad para su profesionalización según relata un exalumno de la carrera: "La universidad vino al sector donde se necesitaba, entonces ahí, mucha gente tuvimos esa oportunidad de estudiar" (Graduado I del PAC entrevista, 2014).

Para los profesores, salesianos y dirigentes de las comunidades, el inicio de un programa universitario de formación docente encaminado a maestros comunitarios tenía un profundo sentido político al articularse a las luchas y reivindicaciones por la educación de los pueblos indígenas. El PAC ofrecía la posibilidad del acceso a la educación superior a una población que, por las condiciones históricas y estructurales de exclusión y marginalidad, de otra manera "jamás hubieran siquiera soñado tener acceso a esta formación” (Profesora B UPS, entrevista, 2015), convirtiéndose así en un programa de formación de educadores imbricado con el proyecto político-étnico de las nacionalidades indígenas, en el que la educación superior es un objetivo estratégico (Farfán, 2008, p. 287).

La pertinencia del programa educativo está constantemente marcada por las tensiones entre necesidades educativas de los sujetos que se educan y la institucionalidad universitaria. Así, la articulación entre las necesidades de los estudiantes, la organización indígena y el proyecto educativo será el desafío constante al interior de la Carrera de EIB, tal como se referencia más adelante.

A continuación, se hace un recuento de la trayectoria de la carrera en el que se pueden distinguir tres momentos relevantes: (Castro, Conejo, Iza, Laso, \& Puente 2009; Reyes, 2006; Farfán, 2008; Cuji, 2011; Granda \& Iza 2012; Villagómez, 2016):

- El inicio, entre 1994 y 2003, conocido como la Universidad en el Páramo. El aporte más significativo de este primero momento fue la posibilidad de contar con un programa de educación superior "propia” y en territorio que permitía de alguna manera dar respuesta a 
las condicionantes socioeconómicas, contextuales, geográficas y a las necesidades educativas de los estudiantes, posibilitando el acceso a la universidad de un sector poblacional históricamente excluido.

- Un programa en crecimiento (de 2003 a 2010). Para el año 2003, el PAC sale del Zumbahua y se establece en ciudad de Latacunga. En 2004 finaliza el convenio con el SEIC "buscando mayor institucionalización y una apuesta por lo pedagógico en una vinculación directa con la UPS" (Farfán, 2008, p. 288). En términos generales, este segundo momento se caracteriza por el crecimiento del programa, tanto por la ampliación de la oferta hacia otros sectores de la población indígena y mestiza de sectores populares, como también por la apuesta por el mejoramiento académico y su organización e infraestructura. En este período la carrera se amplía geográficamente a otras provincias, además de Cotopaxi, se abren los puntos focales en Imbabura, Bolívar, Chimborazo, Morona Santiago y Pichincha.

- Tercer momento, entre 2010 hasta 2018, el programa académico continúa con su trabajo en territorio, aunque con una nueva organización debido a las disposiciones legales de la autoridad de educación superior del país (LOES, 2010). Los puntos focales se denominan centros de apoyo y cuentan con mayor infraestructura tecnológica. La actividad educativa se realiza ya no solamente en las poblaciones donde se encuentran los centros de apoyo sino principalmente en Quito, concentrándose los encuentros presenciales en el Campus Sur de la UPS. Para facilitar la permanencia de los estudiantes en Quito, se cuenta con el apoyo de la Residencia Intercultural, donde los estudiantes con menos recursos pueden hospedarse (Di Caudo, 2014). Para la mediación pedagógica y la gestión del aprendizaje, se implementan los ambientes virtuales de aprendizaje (AVAC) en plataforma moodle, facilitando así el acceso de los estudiantes a los recursos educativos y al apoyo de los docentes.

Finalmente, en el año 2014 se inicia un proceso de rediseño curricular de la carrera de educación de la UPS. El nuevo diseño curricular de la carrera de EIB se implementa en 2018 con una propuesta en modalidad virtual. Esta modalidad exige la producción de materiales y recursos 
específicos para el programa académico y el uso de textos y materiales de apoyo pertinentes a la modalidad, a los destinatarios y a la especificidad del curso. El desarrollo de las actividades educativas se apoya con clases virtuales en tiempo sincrónico, tutorías y encuentros presenciales en territorio y foros, tutorías, chats y mensajería en el AVAC en tiempo asincrónico. La modalidad virtual permite el acceso de un público más amplio y de otros pueblos indígenas además del pueblo kichwa y achuar al que tradicionalmente atiende la carrera.

Un elemento importante a resaltar y que será fundamental en el desarrollo de la vida académica de la Carrera de EIB es la permanencia de las coordinaciones de los centros de apoyo en territorio que se caracterizan por ser espacios importantes de relación entre la institución de educación superior y las demandas de los contextos, decisión que contribuye como se explicará más adelante a dotarle de pertinencia al proyecto educativo.

Actualmente la Carrera cuenta con más de mil estudiantes graduados (UPS, SNA, 2018) con una alta empleabilidad, tanto en actividades vinculadas a la educación y a la docencia, así como cargos directivos en las comunidades de origen y de representación política (Seguimiento-UPS, 2017).

\section{El currículo para la formación docente intercultural bilingüe}

Para Sacristán (2010) el currículo en su significado más sencillo es "el contenido cultural que las instituciones educativas tratan de difundir en quienes las frecuentan, así como los efectos que dicho contenido provoque en sus receptores" (p.12). El currículo por tanto es más que los planes de estudio, porque es un instrumento de naturaleza social, histórica y política. Entonces, siguiendo a De Silva (1999), el currículo no es neutro, privilegia un tipo de conocimiento sobre otros.

La creación del PAC en 1994, como en el caso de todo proyecto educativo formal requería contar con un currículo que permita la concreción de la intencionalidad educativa inicial. El diseño curricular de la carrera fue aprobado en 1995 y revisado en el año 2005. El último rediseño se da en 2017. 
A continuación, realizamos una revisión de los elementos declarados en los documentos curriculares, que dan cuenta de la relevancia de la propuesta educativa que se orienta a una formación específica y especializada para docentes de EIB y que, en el marco de su intencionalidad explicita y privilegia conocimientos, actitudes y valores, alineados con el enfoque intercultural de la educación.

\section{El currículo de 1995 y 2005}

En la justificación del diseño curricular inicial se presentan algunos elementos que dan cuenta de una propuesta educativa que busca atender a la formación de docentes para un sector específico de la población ecuatoriana, la población indígena y la EIB. En relación con los procesos de colonización y subalternización del pueblo indígena, el proyecto educativo presenta una intencionalidad que no se centra únicamente en la formación de docentes de aula y su capacitación didáctica, sino que hacen referencia al desarrollo de pedagogías diferentes (Arroyo, 2012).

Enlistamos a continuación algunas de los elementos caracterizadores de una formación docente diferenciada y especializada para la EIB que delineará luego las decisiones de las concreciones microcurriculares (Villagómez, 2016).

- Ser un instrumento de liberación: mediante procesos educativos que propicien la "liberación de todos los condicionamientos externos e internos que nos enajenan para lograr ser idénticos, ser uno mismo, ser dueño de uno mismo" (UPS-SGU, 1995, p. 103).

- Posibilitadora de autoconciencia y autovaloración a través de "la valoración de su pasado, su sabiduría, su civilización y sus valores” (UPSSGU, 1995, p. 103).

- De inspiración comunitaria: la educación en la reciprocidad para la comunitariedad debe constituirse en una praxis educativa permanente que va desde el compartir la "tonga" hasta la capacidad de escuchar respetuosamente las opiniones del más pequeño de los alumnos" (UPS, 1995, p. 103). "El desarrollo de las capacidades para decidir en función de las necesidades comunes” (UPS-SGU, 1995, p. 104). 
- Ser una herramienta de superación de la pobreza: Educación productiva. La educación intercultural, a nivel superior, debe constituirse en un espacio tiempo en el que la producción de sabiduría, ciencia y tecnología debe ir estrechamente vinculadas a las acciones comunitarias, autogestionarias y productivas (...) (UPS-SGU, 1995, p. 105).

- Posibilitadora de vinculación de los saberes y conocimientos indígenas con los conocimientos de la cultura occidental.

El pueblo indígena posee una sabiduría milenaria, no escrita, que debe ser sistematizada, profundizada y que sin lugar a dudas ha de constituirse en un verdadero aporte, y en una alternativa frente al cientificismo. Por otra parte, la ciencia y la tecnología occidental deben ponerse al servicio de una vida digna para el pueblo indígena (...). (UPS-SGU, 1995, p. 105).

- Constructora de interculturalidad. Los documentos de revisión del la Carrera (UPS, 2005) plantean procesos educativos que aporten a la "construcción social de los pueblos, transitada por la urgencia de que lo indígena recupere su condición de portador de una civilización -la andina- y el mestizo reconozca su identidad reconociendo fundamentalmente, sus raíces andinas" (UPS, 2005).

- Bilingüe, porque desarrolla procesos formativos que fortalecen el dominio comunicativa y académico de la lengua kichwa o achuar y el español. No hay que olvidar que la enseñanza de la lengua indígena constituye uno de los pilares fundamentales del proceso educativo de la EIB por cuanto busca desarrollar habilidades y destrezas en el uso de la lengua y fortalecer la identidad cultural del pueblo indígena.

En correlación con lo expuesto, los objetivos generales del proyecto curricular se orientan a una formación no solo académica, la revalorización de la lengua, la cultura y los conocimientos propios, además de la formación política de los de docentes para la EIB: 
1. Formar, capacitar, profesionalizar docentes identificados con la realidad socioeconómica, política y cultural de las nacionalidades indígenas, que dominen los conocimientos y técnicas en los campos educativo y productivo del proyecto histórico de los pueblos, especialmente indígena (UPS, 2005).

2. Sistematizar, dinamizar y actualizar la sabiduría, la ciencia y la tecnología en función de la dignificación del hombre, especialmente de las nacionalidades indígenas (UPS, 2005).

3. Desarrollar un proceso educativo para-escolar a nivel superior que esté al servicio de las comunidades, organizaciones, instituciones y dirigentes y que se caracterice por responder permanente, sistemática y progresivamente a los requerimientos de formación socio-política de la población indígena (UPS, 2005).

Así mismo, las intencionalidades pedagógicas de las asignaturas previstas en el currículo de la carrera también dan cuenta de la especificidad y especialidad de la formación de docentes para la EIB. El siguiente cuadro resume algunos de los elementos relevantes de los descriptores de cinco asignaturas del proyecto curricular (Villagómez, 2016, pp. 253-254).

Pedagogía Intercultural: La asignatura trabaja la compresión de interculturalidad y de la pedagogía desde una perspectiva intercultural, las experiencias de educación intercultural en Ecuador y en América Latina y sus concreciones curriculares como en lo que tienen que ver con las aplicaciones didácticas y en los contextos escolares y de aula. Realidad Nacional e Indígena: Se trabaja la realidad del país y de los pueblos indígenas en el contexto nacional y en el marco de la constitución del Estado nacional, la herencia colonial y la colonialidad. Así mismo, la realidad del país y de la población indígena en el marco de los contextos neoliberales y posneoliberales. Se centra en el análisis crítico de la situación del indio y sus históricas condiciones de exclusión económica y social. 
Cosmovisión Andina: La comprensión de las relaciones conceptuales del territorio y del manejo de la realidad, la simbología y ritualidad; la cosmovisión como estructura social que permite la identificación (calendario agrícola, astronómico, festivo y religioso); la visión política de las comunidades, los saberes. Los aportes a la sociedad nacional y la democratización de la sociedad y la construcción del Estado plurinacional.

Historia de la Educación y Experiencias de la EIB en el Ecuador: Reflexiona temáticas fundamentales relacionadas con la educación indígena del Ecuador y de educación propia, sus aportes a las condiciones de vida de los pueblos. Así mismo discute los procesos de institucionalización de la educación indígena en el Ecuador, la oficialización de la EIB y los aportes desde la educación a la interculturalidad y a la sociedad en su conjunto.

Lengua indígena o el Kichwa: Estas asignaturas se orientan al fortalecimiento de la lengua materna, a su revalorización, recuperación, los desarrollos para el uso cotidiano, académico, educativo, literario y la investigación del idioma. En este grupo de asignaturas se encuentra el Kichwa I, Kichwa II, Kichwa III y Lingüística Kichwa.

\section{El currículo de 2017}

Como ya se ha mencionado antes, en el año 2014 se inicia en el Ecuador un proceso de rediseño curricular de las carreras de educación bajo las directrices del CES (Consejo de Educación Superior), resultante de los lineamientos de la política educativa nacional. Como parte de la ejecución de la política pública la Comisión Especial del CES, presenta una Propuesta Genérica de las Carreras de Educación (CES, 2015). Este documento curricular de tipo prescriptivo centra la formación docente en la práctica y en la investigación desde la práctica; se reconoce como interdisciplinar e integrador y para ello incluye en su diseño, diferentes asignaturas que aglutinan contenidos de diferentes disciplinas y el desarrollo proyectos integradores. Por su carácter prescriptivo, el currículo genérico orienta en gran medida la estructura curricular y sus componentes. Su carácter homogeneizador, rígido y predefinido en más de un $80 \%$ limitaba 
la posibilidad de incorporar en la malla curricular asignaturas específicas y mucho menos el contar con una propuesta educativa diferenciada. "En un momento pensamos que ni las lenguas indígenas iban a entrar en la nueva malla curricular" (Profesor B, comunicación, 2019).

Sin embargo, luego de un largo proceso de diálogo y negociación académica de más de dos años con las instancias de control de la educación superior del país, se logró la aprobación del nuevo currículo, manteniéndose la especificidad y especialidad de la formación de docentes con pertinencia cultural y lingüística para la educación intercultural, según se constata en los elementos curriculares de nivel meso y micro que se citan a continuación:

\section{Objetivo general}

Formar profesionales de la educación para diseñar y desarrollar propuestas educativas con pertinencia social, cultural y lingüística que contribuyan al desarrollo de los pueblos y nacionalidades indígenas y a la construcción de una sociedad intercultural.

\section{Objetivos especificos}

Al conocimiento y saberes:

- Formar profesionales de la educación que dominen los principales modelos pedagógicos y las teorías del aprendizaje que los fundamentan, así como los contenidos centrales de las diferentes áreas de conocimiento y sus didácticas específicas.

- Formar profesionales de la educación que manejen los principales enfoques de la educación intercultural bilingüe y conozcan las experiencias más importantes impulsadas en América Latina y el Ecuador.

- Formar profesionales de la educación que tengan dominio de su lengua materna y el español, tanto a nivel oral como escrito, y que estén en condiciones de impulsar procesos de educación bilingüe. 
A la pertinencia

- Formar profesionales de la educación con capacidad para diseñar, ejecutar y evaluar propuestas educativas institucionales y de aula con pertinencia social, cultural y lingüística.

- Formar profesionales de la educación con capacidad para retroalimentar su gestión y práctica docente por medio de procesos de investigación acción.

A los aprendizajes

- Formar profesionales de la educación a través de un proceso simultáneo de teorización de la práctica y experimentación de la teoría.

- Formar profesionales de la educación a través de ambientes virtuales que propicien el aprendizaje autónomo y colaborativo.

- Formar profesionales de la educación a través de uso equilibrado de la lengua materna de los estudiantes y el español, y la utilización de recursos y materiales educativos en ambas lenguas.

A la ciudadanía integral

- Formar docentes proactivos en la construcción de una sociedad intercultural, con equidad de género y respetuosa con el ambiente.

- Formar docentes que incentiven la participación ciudadana con miras a la construcción de una sociedad equitativa e inclusiva.

Así mismo, la carrera mantiene en el currículo de 2017 algunas asignaturas que se contemplaban también en el currículo de 1995 y 2005 e incorpora otras que dotan de mayor especialidad a la formación del docente de EIB: El diseño microcurricular expresa la preocupación por proporcionar la pertinencia que la formación exige, tal como se evidencia en los resultados de aprendizaje de algunas de las asignaturas que citamos a manera de ejemplo:

Historia de los pueblos y nacionalidades indigenas:

Analizar las condiciones de vida y estructuras de organización económica, política y cultural de la población indígena antes de la llegada de los españoles. 
Comprender los cambios que la población indígena vivió con el proceso de conquista y la institucionalización del orden colonial.

Explicar la forma como la colonialidad se mantuvo e institucionalizó en la época republicana y las implicaciones que aquello ha tenido para la población indígena.

Discutir las demandas y luchas de la población indígena a lo largo de la historia y sus principales logros. Evidenciar el papel de las mujeres en estas luchas

\section{Cosmovisión indígena:}

Explicar los principios fundamentales de la cosmovisión indígena. Discutir y valorar los aportes de la cosmología indígena y su correspondencia con las actuales prácticas de las comunidades.

Discutir y comparar la concepción indígena y occidental sobre el ser humano y su misión en el universo.

Analizar y valorar los aportes de la ética de los pueblos indígenas para la construcción de una sociedad intercultural y del "buen vivir" (Sumak Kawsay).

Diversidad cultural y lingüística

Comprender y valorar el carácter diverso del Ecuador, desde el punto de vista cultural, lingüístico y de género.

Analizar los elementos centrales de la cultura y lengua de los pueblos indígenas y de la población afrodescendiente, montubia y mestiza.

Discutir las relaciones de poder que perviven entre los diferentes grupos culturales en la actualidad y las asimetrías que existen a nivel de acceso a bienes y servicios.

Debatir sobre las diferentes formas en las que se expresan las relaciones de género en el mundo indígena ecuatoriano.

\section{Modelos y Experiencias de Educación Intercultural Bilingüe}

Analizar los procesos educativos hegemónicos impulsados en el país y su impacto en la población indígena.

Analizar las experiencias de EIB más relevantes en el país. Identificar y diferenciar los modelos de EIB que se han impulsado en el país y la Región Andina. 
Explicar los fundamentos teóricos de la EIB.

Lengua Indígena I:

Reconoce el origen y la importancia del uso de las indígenas en el Ecuador. $(\ldots)$

Educación y bilingüismo

Planificar y aplicar en aula, procesos metodológicos tendientes a desarrollar el

bilingüismo.

Además de las asignaturas citadas, todas las materias del campo de la didáctica general y específicas se traban en perspectiva y enfoque intercultural, tal como se señala en el mismo documento curricular (Didáctica General, Ciencias Sociales y su Didáctica con enfoque intercultural, Matemática y su didáctica con enfoque intercultural, Ciencias Naturales y su Didáctica con enfoque intercultural) (UPS, SGA, 2017).

El diseño y desarrollo curricular, como se explicita guarda relación con un enfoque educativo intercultural. En ambos proyectos académicos, tanto el inicial, como el 2017 el esfuerzo se centra en la comprensión de las necesidades educativas de los estudiantes y sus contextos, sus espacios laborales, la reflexión educativa y el ejercicio de la docencia para la educación intercultural bilingüe. Para el trabajo educativo se opta por metodologías "que propiciarán la incorporación del diálogo de saberes ancestrales, cotidianos y tradicionales, de inclusión, diversidad y enfoque de género" (UPS, SGA, 2017).

Se trata de un diseño curricular que incluye un fuerte componente de práctica y de vinculación con la realidad; así, las prácticas se realizan en todas las unidades de formación (básica, profesional y titulación) y en cada uno de los semestres, permitiendo al estudiante una cercanía con el hecho educativo y sus contextos, el ejercicio de la docencia y la gestión e intervención, desde la investigación educativa. La práctica incide en la configuración curricular (Gimeno Sacristán, 1991). 


\section{Los desafíos de la práctica y la implementación del proyecto desde la perspectiva de los actores}

Aunque hasta aquí se ha hecho referencia al currículo oficial o prescriptivo, no hay que perder de vista el currículo real o vivido Stenhouse (1991) que es aquello que efectivamente ocurre en el espacio educativo y que estará condicionado por las propias características de los sujetos que se educan y, por supuesto, de los que enseñan y de los contextos. Implican 'negociaciones' en términos pedagógicos entre maestros y estudiantes y las demandas donde el hecho educativo ocurre. Así, la comprensión de la ejecución de un proyecto académico no se remite exclusivamente a explicar cómo se concreta un currículo de manera funcional, sino que es necesario considerar los desafíos reales que esa concreción demanda.

A continuación, recuperamos los testimonios de los docentes, estudiantes y directivos de la carrera, (Villagómez, 2016) que dan cuenta de estos retos en su propia voz. La revisión de los testimonios ha sido organizada considerando los componentes del diamante curricular De Zubiría (2006), por un lado, particularmente: fines, contenidos, métodos y medios del proceso de interaprendizaje; y por otro lado, los fines de la educación intercultural y la praxis de pedagogías interculturales:

La educación intercultural como fundamento de la práctica

“(...) la interculturalidad es de relaciones interpersonales, de relaciones intercomunitarias, de valoraciones mutuas. Por ahí pasa la interculturalidad, en el fondo" (Salesiano A, entrevista, 2014) y en este sentido los espacios educativos deben propiciar ese conocimiento y esas valoraciones mutuas. De acuerdo con la perspectiva de una de las profesoras de la carrera, esto será posible solo si se reconocen e interrogan las históricas relaciones de desigualdad y exclusión de los grupos subalternos, entre ellos los pueblos indígenas. Así, atender desde la educación a la construcción de relaciones menos desiguales en la sociedad y a la valoración del otro implica comprender de manera crítica que:

(...) en los mismos procesos educativos existen relaciones inequitativas, por eso un intento de abrir canales, yo veo que es posible pero con 
toma de conciencia clara de las posturas y de los niveles de poder, de lo inequitativo, a partir de ese reconocimiento, es posible integrar ese diálogo y eso en perspectivas de largo plazo para lo que se requiere esfuerzos serios, sistemáticos, organizados de todos los actores, con amplia participación de los estudiantes, con el empoderamiento de los mismos estudiantes. (Profesora A, entrevista, 2015)

A partir de esta reflexión crítica se puede propiciar desde la educación las condiciones para fomentar el diálogo e interacciones menos inequitativas entre los sujetos y también entre los conocimientos y los aprendizajes, tal como se expresa en los siguientes testimonios:

- $\quad$ "Se debe enseñar en las dos lenguas, en kichwa y en castellano (...) y los niños expresarse libremente en su propio idioma” (estudiante C, entrevista, 2016);

- "debe ser una educación contextualizada, basada en la realidad donde vivimos, partiendo de nuestros saberes, experiencias, identidades" (estudiante E, entrevista, 2016);

- que "contemplen en los contenidos curriculares, los conocimiento y sabiduría de diferentes culturas" (estudiante H, entrevista, 2016);

- “y que exija el respeto de todas las creencias, tradiciones, culturas, lenguas y los derechos, exija también la igualdad sin ninguna discriminación" (estudiante I, entrevista, 2016).

- "Los contenidos deben elaborase basándose en la realidad sociocultural del niño y en dos idiomas" (estudiante J, entrevista, 2016);

- debe trabajar desde "nuestros conocimientos ancestrales" (estudiante E, entrevista, 2016) (Villagómez, 2016, p. 210).

Finalmente, desde la perspectiva de los sujetos “(...) (la interculturalidad) es práctica, no solo es decir” (Graduada PAC B, entrevista, 2014) y la educación intercultural es el camino para ese logro. El desafío de la 
carrera tiene que ver con generar los espacios de reflexión y agencia para que la interculturalidad se lleve a la práctica en los procesos educativos y para que los estudiantes/maestros sean capaces de generar experiencias educativas con enfoque intercultural en sus comunidades y contextos.

Sobre los fines: ¿Para qué enseñar y aprender?

De acuerdo con la perspectiva de los actores la intencionalidad de la carrera está relacionada con una formación docente que enfatiza en el fortalecimiento identitario, cultural y lingüístico de los estudiantes y que les posibilite trabajar en sus propios contextos y comunidades desde una perspectiva intercultural en diversos espacios educativos, áulicos, escolares y comunitarios.

(...) es por eso que, ya desde esos años de finales de los años ochenta se empieza a buscar una alternativa de formación Superior para formar los docentes. Pero era para formar un docente al servicio de la comunidad con una perspectiva liberadora, crítica, con fuerte identidad cultural, al servicio de las comunidades. Ese tipo de docente se quería; que fortalezca y que sea parte de la organización indígena. No aparte (...). (Salesiano A, entrevista, 2014)

Sobre los contenidos: ¿Qué enseñar y aprender?

En los testimonios se explicita la necesidad de incorporar entre los contenidos de aprendizaje el conocimiento y valoración de la lengua, cultura e historia propia. Además de los conocimientos y saberes propios y los occidentales, según se afirma en el diálogo con profesores y estudiantes de la Carrera:

Entonces el gran reto (...) si no trabajas en la línea de la autovaloración en términos individuales y colectivos, muy poco vas hacer... en ese marco la entrada de habla de nosotros, de lo propio, de recuperar la propia historia, creo que si es necesario en un momento... para ese trabajo más formativo. (Profesor B, entrevista, 2016)

(...) la recuperación del conocimiento indígena y la valoración se empiezan a dar con la recuperación de los cuentos, de los mitos indígenas 
y que se empieza a convertir en contenidos educativos. La recuperación de la historia, o sea, una relectura de la historia indígena ya no desde las lecturas oficiales... las historias oficiales. Al recuperar los líderes indígenas, historias comunitarias, la historia de los dirigentes, la historia de la lucha por la tierra. Junto con eso, el conocimiento que se llamaba occidental; las Ciencias Naturales, la Matemática. Entonces siempre estuvo esa tensión entre cómo la escuela no se convierte en quien va eliminando el conocimiento indígena y como también le va dando herramientas que necesita. (Salesiano A, entrevista, 2014)

Está bien hacerles conocer las teorías occidentales (...), pero hay que hacerles conocer contrastando con la metodología propia... partir de lo que ya tenemos... en algunos casos estamos más adelantados, por otros caminos. No es la única ciencia que hay. Hay varias ciencias (...). (Profesor, F, 2015)

La educación intercultural "necesita fortalecer la identidad cultural, las lenguas y la organización de los pueblos y nacionalidades". (Estudiante I, entrevista, 2016)

Sobre los métodos y los medios: ¿Cómo y con qué enseñar y se aprender? El desarrollo del proyecto educativo pone en evidencia la necesidad de incorporar métodos, formas y estilos propios de aprendizaje, desde las necesidades de los sujetos y sus contextos.

Bueno, yo al menos que formo del primer grupo, digamos, del PAC que era en ese entonces; para nosotros, al menos, ha sido una oportunidad. Porque aparte de trabajar, nosotros tuvimos la oportunidad de estudiar en un sistema totalmente distinto. Donde las enseñanzas que nos daban siempre nos enfocaban a la realidad donde estábamos. O sea, la realidad donde nosotros vivimos. Y además de eso, uno de los objetivos era que se fortalezca y se mantengan los valores culturales. Siempre, decía, por ejemplo, en Sigchos en Chugchilan, ¿cómo vivía la gente? ¿Qué valores tenían? O sea, siempre las tareas que nos enviaban, siempre enfocado a la realidad, sin dejar de lado la cuestión científica, también. (Graduado A, entrevista, 2014)

(...) lo comunitario, lo cercano, lo vivencial, sin perder esto, tenemos que formar a nuestros docentes. Siempre tenemos que tener enfatizar, lo pedagógico tenemos que relacionar con lo comunitario. Relacionar con la comunidad. Lo didáctico, relacionar con la comunidad, la inter- 
culturalidad, la cultura, la parte curricular. Yo creo que tenemos que relacionar siempre estar enfatizando. O sea, para no irnos desvinculando. (Profesora C, entrevista 2014)

Así mismo, la concreción del proyecto educativo exige un constante vínculo con los contextos y la pertinencia del proyecto educativo, su relación con los proyectos educativos propios y de organización del pueblo indígena. De allí que la presencia del programa en territorio a través de la figura de los centros de apoyo es un elemento fundamental en el desafío siempre vigente de dotarle de pertinencia a la carrera.

Los coordinadores de los centros de apoyo son los actores que vienen de una red que también son claves en lo que sería una relación orgánica entre carrera y organizaciones y comunidad. A, L, T, C, tienen excelentes relaciones con las organizaciones, con los gobiernos locales, pero también con los distritos, municipios. Son redes invisibles, que dan vida al proyecto, son formas propias de organización andina y que en definitiva ayudan a mantener contacto con tierra, ayuda a alimentar la carrera siempre. No entenderías el éxito de la carrera y la pertinencia sin estas relaciones más informales (...). (Profesor B, de la carrera, entrevista, 2016)

Sobre las pedagogías interculturales

La pedagogía y la educación su objeto de estudio, hacen referencia a articulaciones entre experiencias, saberes y conocimientos, la sociabilidad y la subjetividad como construcciones y producciones. Como práctica social está históricamente situada en una coyuntura o en un orden civilizatorio (Puiggrós \& Marengo, 2013, p. 21). Implica también esas relaciones entre los sujetos que intervienen en el proceso educativo, sus contextos y los contenidos curriculares (saberes, hacerse y, actitudes y valores) que se producen en la praxis de la concreción curricular y educativa y que dan respuesta a esas realidades educativas situadas.

Hace parte del proyecto educativo de la carrera, el anhelo y construcción y praxis educativas que al interrogar los modelos tradicionales por ser insuficientes o contrarios a los fines de la educación intercultural generen respuestas político-pedagógicas alternativas o "insumisas" y creen los espacios para que esa otra educación sea posible (Medina, 
2015). Se trata de reflexionar en pedagogías interculturales: de "concientización de la opresión", la discriminación, el racismo y también de liberación (Freire, 1970), de autovaloración, de reafirmación y recomposición identitaria; del acercarse al conocimiento del otro-, pero para retornar empoderado y para aportar a la comunidad y a la educación propia e intercultural; de recuperación, sistematización, uso y vitalidad de la lengua y los conocimientos propios, pero también del aprendizaje del conocimiento occidental; son pedagogías de solidaridad y de sororidad y también del afecto.

Se transcribe a continuación tres testimonios que dan cuenta de los desafíos pedagógicos del programa académico. El primero de ellos pone de manifiesto el tema de autovaloración cultural, identitaria y lingüística, el segundo da cuenta de las relaciones de los sujetos en el proceso educativo de la carrera y finalmente, el tercero tiene que ver con la incidencia en los contextos educativos propios:

(...) yo soy de Riobamba ... mi madre es indígena, utiliza el 'anaco', 'la bayeta' y somos del Cantón Colta. Pero, la verdad a raíz, de que ella pasó por una discriminación, como decirnos; ella no podía utilizar el anaco, la discriminaban por su vestimenta, inclusive por su forma de... hablar, por su lenguaje. Entonces mi mami tomó la opción de 'aculturarnos', diríamos, a lo que es los mestizos.

Por eso yo visto lo que es ahora el pantalón. Pero realmente esta no es mi vestimenta, es una vestimenta que se acopló a mí, porque mi madre dijo, 'no quiero que sufras lo que yo sufrí', por una parte. Y el leguaje digamos... los maestros deberíamos aprender, es porque, por ejemplo, yo digamos, mi abuelita es puro Kichwa, ella no sabe el Español, con ella solo hay que relacionarse solo con el Kichwa. Entonces, yo sabía, como se dice, mi mami me decía, 'tú no tenías el kichwa mezclado, sino el kichwa puro'. Entonces, (con mi experiencia, ¿no?) me fui al Jardín (de infantes) me acuerdo, y yo hablaba el kichwa, pero mi maestra no me entendía. Entonces, mi maestra, digamos era..., de acá, y era hispana, y yo a veces le pedía permiso, así al baño o situaciones que a veces uno necesitaba. Imagínese, yo lloraba, le decía que me de permiso para ir al baño, en mi idioma y mi maestra, no entendía... 
Yo fui forzada, de niña...La verdad yo me estaba olvidando mi idioma... medida de lo que... fui, o sea, forzada, ¿no? Iba en la casa, mi maestra..., (mi mamá mi papá se fueron jóvenes a trabajar y yo estuve a cargo de mi abuelita y mi abuelita siempre hablaba el kichwa, yo también hablaba solo en kichwa, pero a raíz de lo que no me entendía (mi maestra) y no me daba a entender, o sea de parte y parte no nos entendíamos, ella empezó como a investigar mi maestra.

(...) yo en realidad, en realidad, fortalecí más este idioma (kichwa), en mi persona (como indígena), es aquí en la Universidad (...). (Graduada B del PAC, entrevista, 2014)

En el aula había esa diversidad. Entonces, por ejemplo, yo... en mi aula había más compañeros que hablaban kichwa, entonces hay un profesor de kichwa y se entendían. Hasta por broma se comunicaban y hablaban. Pero había otros profesores que no hablaban. Entonces hablábamos en castellano mismo... a veces algunos compañeros se equivocaban en las letras y les daba ánimo; les decían ustedes pueden redactar bien, hacer bien. Porque era importante que aprendan para relacionarse. (Estudiante B PAC, entrevista, 2014)

Para eso vine acá, a la universidad, porque yo estoy dentro de una comunidad y llevarme, lo que yo aprendo aquí, llevo a compartir en mi comunidad, tanto en mi trabajo, con los padres de familia, con los profesores igual en mi comunidad. (Estudiante F, PAC, entrevista, 2014)

\section{Consideraciones finales}

La carrera de EIB, constituye un proyecto educativo emblemático no solo en el Ecuador sino también en la región. Su especificidad es la educación intercultural bilingüe y la formación de docentes para la EIB. Su fin último es aportar desde la educación a la construcción de una sociedad intercultural.

Pensar en una docencia para la educación intercultural bilingüe exige como se ha puesto en evidencia no solo el desarrollo pedagógico y curricular alternativo, crítico y creativo, sino la puesta en marcha de procesos educativos que exigen concreciones curriculares metodológicas y didácticas también diferenciadas, críticas y pertinentes a las necesidades y demandas de los sujetos y de los contextos. 
La formación de profesorado para la EIB es un campo relativamente nuevo dentro de los debates más actuales sobre la profesión docente; implica consideraciones ético-políticas, pero también disciplinares además de pedagógicas y metodológico-didácticas que atiendan a las exigencias prácticas que el ejercicio de la docencia en contextos de diversidad cultural y lingüística requiere.

La revisión de la experiencia concreta de la carrera de EIB de la UPS, como programa de formación docente pone en evidencia la necesidad de interrogar la matriz colonial de la sociedad ecuatoriana al considerar las inequitativas relaciones de poder entre los sujetos que hacen parte de los procesos educativos, los objetos y conocimientos que en ellos se incluyen. Junto con ello, se impone la necesidad de recuperar, sistematizar en incluir en los procesos educativos los conocimientos, la lengua y la cultura de los pueblos indígenas. Cobra importancia también el reto de incluir otras apuestas curriculares y pedagógicas como herramientas para atender a los fines de la educación intercultural e intercultural bilingüe y a la formación de su profesorado. A nuestro entender es en lo pedagógico donde entra en juego el éxito, pertinencia, efectividad y eficacia de cualquier proyecto educativo.

Finalmente se insiste en la necesidad de abrir el debate académico-pedagógico sobre la formación del profesorado y la docencia para la EIB. La revisión de la experiencia de la carrera de EIB de la UPS realizada en este trabajo es una invitación a profundizar en futuras investigaciones en la comprensión de los procesos educativos trabajados durante estos años, su incidencia y aportes a la educación intercultural e intercultural bilingüe del país.

\section{Referencias bibliográficas}

Arroyo, M. (2012). Outros sujeitos outras pedagogías. Belo Horizonte: Editorial Vozes.

Castro, G., Conejo, N., Iza, A., Laso, P., \& Puente, T. (2009). La Universidad en el páramo. El Programa Académico Cotopaxi. Quito: Abya-Yala.

CES. (2015). Rediseño curricular de las carreras de educación del Ecuador. Ecuador. 
Cuji, L. (2011). Educación superior e interculturalidad. FLACSO Sede Ecuador. Recuperado de: http://bit.ly/2M0RksI

De Silva, T. (1999). Documentos de Identidad Una introducción a las teorías del currículo. Belo Horizonte: Autêntica Editorial. Recuperado de: http:// bit.ly/2Z0VZyD

De Zubiría, J. (2006). Los modelos pedagógicos (Segunda). Bogotá D.C.: Aula Abierta. Recuperado de: https://goo.gl/T5KcA6

Di Caudo, M. (2014). Estudiantes inteculturales en la Universidad. Un relato colectivo desde Ecuador (Primera). Cuenca: Abya-Yala. Recuperado de: http://bit.ly/2LONIuH

Farfán, M. (2008). Experiencia del Programa Académico Cotopaxi, Formación en Educación Intercultural Bilingue de la Universidad Politécnica Salesiana del Ecuador. In Diversidad Cultural e interculturalidad en Educación Superior en América Latina. Caracas: IESALC-UNESCO.

Freire, P. (1970). Pedagogía del oprimido. Buenos Aires: Siglo XXI Argentina Editores, S.A.

Granda, S, \& Iza, A. (2012). “Los salesianos, la educación superior y los pueblos indígenas. El caso del Programa Académico Cotopaxi”. In La presencia Salesiana en Ecuador. Perspectivas históricas y sociales. Quito: Abya-Yala.

Granda, S. (2018). Transformaciones de la Educación Comunitaria en los Andes Ecuatorianos. Sophia, Colección de Filosofía de La Educación, (24), 291-311. Recuperado de: http://bit.ly/2LYqf9D

LOES. (2010). Ley Orgánica de Educación Superior. Quito, Ecuador. Recuperado de: http://bit.ly/30yVoVk

Medina, P. (2015). Pedagogías insumisas. Movimientos político-pedagógicos y memorias colectivas de educaciones otras en América Latina. México: Juan Pablos Editor.

Puiggrós, A., \& Marengo, R. (2013). Pedagogías: reflexiones y debates (Primera). Buenos Aires: Universidad de Quilmes. Recuperado de: https://goo. $\mathrm{gl} / \mathrm{joWjSt}$

Reyes, D. (2006). La construcción de la interculturalidad desde las politicas educativas en la experiencia del sistema de formación docente en el Programa Académico Cotopaxi, de la Universidad Politécnica Salesiana. Tesis de Maestría (2010). ¿Qué significa el currículum? Sinéctica, 34, 11-43. Recuperado de: http://bit.ly/2JNDTd2 
Gimeno Sacristán, J. (1991). El currículo: Una reflexión sobre la práctica. 9na edición. España: Morata

Seguimiento-UPS. (2017). Seguimiento de Egresados, Carrera de EIB. Cuenca.

Stenhouse, L. (1991). Investigación y desarrollo del curriculum. Madrid: Ediciones Morata.

UPS-SGU. (1995). Sistema General de la UPS. Cuenca.

UPS. (2005). Licenciatura en Ciencias de la Educación con Mención en Docencia Básica Intercultural Bilingüe. Cuenca. Recuperado de: http://bit.ly/32xRn5e (2018). SNA. Cuenca.

Villagómez, M. S. (2016). Práctica de la Interculturalidad, descolonización y formación docente: El Programa Académico Cotopaxi del Ecuador. Universidad Federal de Minas Gerais. 